\title{
Caring for the Person with Motor Disability: Implications for Nursing
}

\section{Summary}

The rehabilitation of the person with motor disability starts from the hospital and the caregiver plays a fundamental role in achieving the objectives, in this sense it is necessary for the nursing professional to identify some of the elements that are presented during the care relationship; including complementarity, risk prevention, coexistence, fostering independence, providing well-being and caregiver learning, which will contribute to achieving the greatest possible independence of the person with motor disability.

Keywords: Care; Caregivers; Disability

\section{Introduction}

In Mexico there are about 6 million people with some physical or mental disabilities to perform activities of daily living. $40.7 \%$ of people with a disability are between 60 and 84 years of age, $32.8 \%$ are in the adult age between 30 and 59 years and the minor percentage is represented by the population of 85 years and more. It is worth mentioning that two out of ten individuals with disabilities are under the age of 30 [1]. The disability that is most frequently presented to the Mexican population is related to mobility, since about half of the reported limitations refer to walking or moving (58.3\%). The main cause of disability is due to diseases (39.4\%), followed by advanced age (23.1\%); and the limitations due to birth and accident affects almost one-third of the population with disabilities with $16.3 \%$ and $15 \%$, respectively [1].

In situations like this, most family members provide the support, however they are asked for commitment and dedication, since they must invest time to provide care aimed at meeting basic needs. It is for this reason that the participation of the caregiver in the hospital care of the person with motor disability is fundamental to the achievement of the objectives of rehabilitation, their collaboration is paramount, however, nursing professionals must take into account certain considerations, which are broken down below.

Complementarity in care, the nurse and the caregiver develop a social role of formal and informal care in the hospital area, are organized, synergized and empowered with the objective of satisfying basic needs of the person with motor disability, this manifests itself through collaboration, help, teaching, supervision and alliances between the nurse and the caregiver.

The prevention of risks demands that the caregiver perform actions and behaviors aimed at the protection of the person who cares [2], the above in order to avoid complications arising from the vulnerability of the person with motor disability and events originating from medical care, these include the prevention of pressure ulcers, falls and infections associated with health care.

Oolume 9 Issue 6 - 2017
Sandra Hernández Corral1** and Ma.
Guadalupe Ojeda Vargas ${ }^{2}$
${ }^{1}$ Instituto Nacional de Rehabilitación "Luis Guillermo Ibarra
Ibarra", México
${ }^{2}$ Universidad de Guanajuato, Campus Celaya, México
*Corresponding author: Sandra Hernández Corral, Instituto
Nacional de Rehabilitación, Calzada México-Xochimilco No.
289, Colonia Arenal de Guadalupe, Delegación Tlalpan, C.P.
16018, Ciudad de México, México, Tel: 59 99 10 00; Ext:
12219; Email: shcorral@gmail.com
Received: September 27, 2017 | Published: October 06,
2017

Living with the person with motor disability causes satisfaction, peace, love, tranquility and feelings found in the caregiver. Similarly, the caregiver responds to the proposals launched by being cared for by providing encouragement and comfort and at the same time establishing new ways of relating to each other, these are shaped by both are in constant interaction and reciprocity, learn from each other and facilitate care [2].

The rehabilitation process involves fostering the independence of the person with motor disability, in which the caregiver plays a fundamental role in maintaining a balance between dependence and independence, so that the caregiver recognizes the need to identify the level of functional independence and its evolution during the process of rehabilitation, in this sense the balance is variable and dynamic, this gives rise to the caretaker a constant search for the right measure that does not stop and that with the passage of time diversifies their activities.

The activities of daily living are the tasks that a person must be able to perform to take care of himself independently, these include personal care that provides well-being, however, people with motor disabilities may have a diminished functional capacity and as a consequence they require partial or total assistance for bathing, dress, food and continence; in this sense the caregiver develops the capacity to identify the degree of help that the caregiver requires and from the above they are able to provide the necessary help that can range from supplementing the activity, provide partial assistance or supervise the activity.

The caregiver is an important variable, their presence with the patient and their affection are fundamental, it is of vital importance that the caregiver has the necessary knowledge to provide the care support. The teaching is provided by the nursing staff and is focused on the satisfaction of basic needs, 
privileging the eminently physical issues. The learning techniques used by caregivers are the observation, explanation, guidance and collaboration of professionals in the execution of care, nevertheless one learns with the person with motor disability so it is essential to consider their specificity and its uniqueness.

\section{Conclusion}

Caring for a person with motor disability: it is the complementarity in the care that enhances the satisfaction of the basic needs of the person; is the practice of risk prevention that will avoid the complications derived from the condition of vulnerability; It is the living with the person with motor disability that provokes satisfaction, peace, love and feelings found in the caregiver; is the living with the disabled person that generates new ways of relating to each other, originated by constant interaction and reciprocity; is where the caregiver and the person with disabilities learn from each other to facilitate care; It is the balance between dependence and independence that allows to maintain a balance between the excess and the defect of the care;
It is the just measure between dependence and independence that will change according to the functional capacity of the person with disability; It is the welfare provided by the caregiver that guarantees the satisfaction of the basic needs of the disabled person; It is the caregiver's education that guarantees the satisfaction of the basic needs of people with disabilities; It is learning through observation, explanation, guidance and collaboration of professionals in the execution of care.

\section{Conflict of interest}

The authors declare no conflict of interest.

\section{References}

1. Instituto Nacional Estadística Geografía e Informática (2010) Principales resultados del Censo de Población y vivienda. INEGI, México.

2. Boff L (2002) El cuidado esencial. Ética de lo humano compasión por la tierra. Madrid, Spain. 